\title{
Primary Lymphoma of the Heart: A Case Report of Surgical Treatment and Review of the Literature
}

\author{
Shuai Liu, ChongLei Ren, Rong Wang, Yao Wang \\ Department of Cardiovascular Surgery, PLA General Hospital, Beijing, China
}

\section{ABSTRACT}

Background: The incidence of heart tumors is the lowest in all known tumors. Primary cardiac tumors are especially rare, with an incidence of $0.056 \%$ according to autopsy reports. The most common type is myxoma, which account for nearly $70 \%-80 \%$ of them. Only $10 \%$ of primary cardiac tumors are malignant, approximately $95 \%$ of which are sarcomas, whereas the primary cardiac lymphomas (PCLs) are merely $1 \%$.

Case Presentation: The present study reported a case of PCL with bilateral renal involvement. The patient received right atrial tumor excision and cardiac pacemaker implantation because of obstruction of the right ventricular outflow tract and third degree atrioventricular (AV) block. Primary diffuse large B-cell lymphoma (DLBCL) was diagnosed through pathological examination; complete remission (CR) was achieved after timely treatment with chemotherapy and autologous peripheral blood stem cell transplantation.

Conclusions: PCL is a very rare disease with highly malignancy. With nonspecific clinical features, the diagnosis is mainly done by histopathological and immunohistochemical staining. At the moment, the most effective treatment is chemotherapy. Palliative surgery may be necessary to correct hemodynamics when outflow is obstructed. The specific location, size, and proximity of the tumor 1 ead to the prognosis. Once the cardiac conduction system was involved, electrocardiography (ECG) showed high AV block.

Received September 15, 2018; received in revised form fanuary 7, 2019; accepted February 26, 2019.

Abbreviations: $A V$, atrioventricular; $C R$, complete remission; $D L B C L$, diffuse large B-cell lymphoma; ECG, electrocardiography; HBs Ag, hepatitis B surface antigen; HCV, hepatitis $C$ virus; HL, Hodgkin lymphoma; HIV, buman immunodeficiency virus; NHL, non-Hodgkin lymphoma; PCL, primary cardiac lymphoma; PET-CT, positron emission tomography and computed tomography; PR, partial remission; $R$-CHOP, rituximab, cyclophosphamide, bydroxydaunomycin, Oncovin, prednisone ; R-EPOCH, rituximab, etoposide phosphate, prednisone, Oncovin, cyclophosphamide, hydroxydaunorubicin; TEE, transesophageal echocardiography.

Correspondence: ChongLei Ren, Department of Cardiovascular Surgery, PLA General Hospital, 100853 Beijing, China; 13301317885 (e-mail: renchonglei301@163.com).

\section{INTRODUCTION}

The incidence of heart tumors is the lowest in all known tumors. Primary cardiac tumors are especially rare, with an incidence of $0.056 \%$ according to autopsy reports [Lam 1993]. The most common type is myxoma, which account for nearly 70\%-80\% of them [Basso 2013; Baikoussis 2015]. Only $10 \%$ of primary cardiac tumors are malignant, approximately $95 \%$ of which are sarcomas, whereas the primary cardiac lymphomas (PCLs) are merely 1\% [Travis 2015]. PCL is pathologically divided into non-Hodgkin lymphoma (NHL) and Hodgkin lymphoma (HL). The clinical manifestations may vary and are attributed to the turmor's location and size and the range of tumor invasion. The diagnosis is difficult, as well as the prognosis is poor [Coiffier 2002]. The patients with PCL often die from rupture of the heart, large-area pulmonary embolism, heart failure, or malignant arrhythmia [Petrich 2011]. In this case report, 1 patient diagnosed with PCL and treated at our clinic is presented.

\section{CASE REPORT}

We report about a 46-year-old man with a history of exertional dyspnea and backache for 1 month prior to hospitalization, along with abdominal distention, diarrhea, and limb edema. The patient had no complaints of fever, chest pain, cough, hemoptysis, expectoration, or paroxysmal nocturnal dyspnea. On physical examination, heart rate was $41 \mathrm{bpm}$; blood pressure, 128/72 mmHg. There was no jugular distention. The apical pulsation was weak, and the boundary of the heart was enlarged; the heart sounds were low and distant, as well as the systolic murmur could be heard in the auscultation area of the tricuspid valve. Laboratory data were as follows: tests for HBsAg, anti-HCV, and anti-HIV were negative. C-reactive protein, erythrocyte sedimentation, liver and renal functions, and blood sugar were all within normal limits. Lactic acid dehydrogenase was 616 IU/L. Electrocardiography (ECG) showed atrial flutter and third degree atrioventricular (AV) block. Chest x-ray showed an enlarged heart. Echocardiography revealed a large circumferential pericardial effusion and an heterogeneous tumor from the free wall of the right atrium entering the right ventricle. The tumor was about $5.0 \times 3.5 \mathrm{~cm}$ in size, was almost closing the orifice of the tricuspid valve, was adhering to the tricuspid valve, and led to severe tricuspid valve insufficiency (Figure, A). A series of whole-body examinations were performed for tumor 


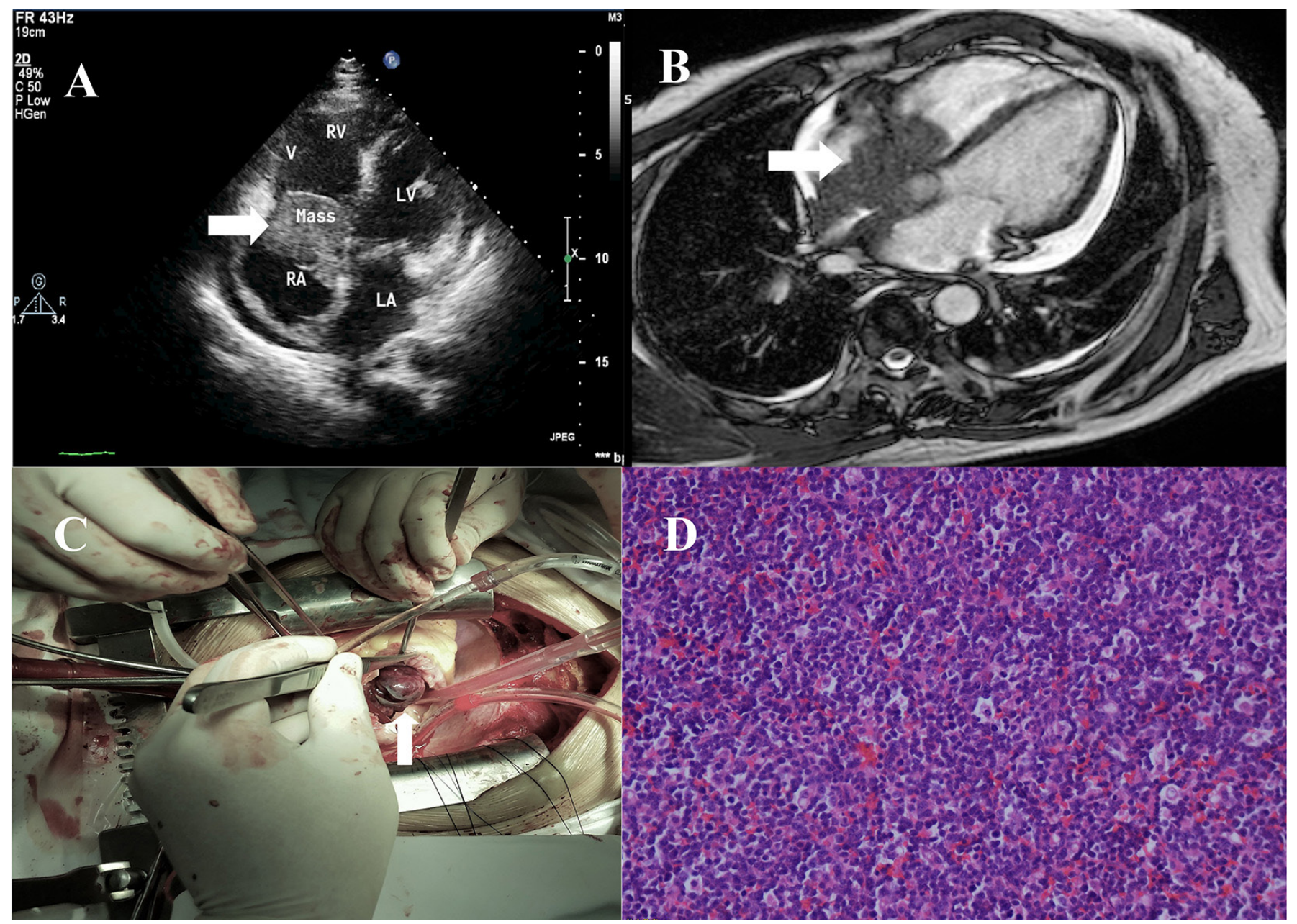

(A), Four-chamber echocardiogram showing a heterogeneous tumor located at the right atrium and having closed the orifice of the tricuspid valve (see arrows). (B), Cardiac magnetic resonance image demonstrating a nodular mass extending into the right ventricle at the level of the atrioventricular (AV) canal, with narrowing of the right AV orifice (see arrows). (C), The large tumor mass during the operation (see arrows). (D), The pathological examination showing a diffuse large B-cell lymphoma (DLBCL).

staging, such as MRI of the heart and MRI gastrointestinal series, CT scan of the heart and whole abdomen, and positron emission tomography and computed tomography (PET-CT). There were infiltrative tumors involving the right atrium, pericardium, bilateral kidneys, and lymph nodes in the right inguinal region and retroperitoneum; the infiltrative tumors in this case were consistent with signs lymphoma (Figure, B). Considering the possibility of right atrial tumors blocking the tricuspid orifice at any time, right atrial tumor excision and cardiac pacemaker implantation were performed. During the operation, we can see the tumor occupied most of the right atrium, blocked the tricuspid valve orifice, and extended to the superior vena cava (Figure, C). The tumor could not be resected clearly, because the junction between the pedicle and the anterior septum of the tricuspid valve and the Koch triangle area were tightly adhered, the invasive site was cauterized, and the epicardial pacemaker was implanted. The pathological examination showed a picture of a diffuse large B-cell lymphoma (DLBCL) (Figure, D). Immunocytochemical stains of the sections of the tumor exhibited large lymphoid cells positive for Ki-67 (+, about 90\%), CD20, Mum-1, Bcl-2, Bcl-6, c-MYC and negative for CD5, CD10, CD30, CD3, CD23, cyclin D1. The following chemotherapy was performed the on 20th day after operation: 6 courses of R-CHOP, 1 course of R-EPOCH and autologous peripheral blood stem cell transplantation were performed, PET-CT showed complete remission (CR). The patient remained free of lymphoma in the follow-up at 1 year.

\section{DISCUSSION}

PCL is extremely rare and often seen in patients with immune deficiencies. There are 2 way to come to a determination of PCL; one is only confined to the heart and pericardium; the final diagnosis can only be made after careful autopsy because lesions in other parts of the body should be taken out [Curtsinger 1989]. The other is first diagnosed as a 
mass of tumor tissue in the heart or cardiac-related symptoms caused by the infiltration of lymphoma, sometimes accompanied with enlargement of mediastinal lymph nodes and pleural exudation [Zaharia 1991; Petrich 2011]. This case belongs to the second; chest tightness and shortness of breath were the main symptoms before hospitalization; and a huge mass filled the right atrium with narrowing of the right AV orifice. PCL is more often found in male adults, although it is also reported in children [Ceresoli 1997]. The pathogenesis of PCL is still unclear. Several studies have reported that infection or immune deficiencies can make the normal lymphoid organization in the heart turn into lymphoma, such as HIV infection, congenital immune deficiencies, EB virus infection, or situations needing solid organ and allogeneic bone marrow transplantation. These pathological types are common in small non-cleaved cell lymphoma, immunoblastic lymphoma, and so on. Although DLBCL accounts for about $80 \%$ of PCLs in patients with normal immune function [Tanaka 2009; Svec 2012], this case is a male immunocompetent patient with DLBCL, which was consistent with the literature.

The symptoms of PCL are nonspecific; any part of the heart may be involved. The main location may exist right atrium, pericardium, or right ventricle, or left atrium, or left ventricle. The symptoms mainly depend on tumor location, size, growth rate, degree of invasion, and friability. It can manifest as pericardial effusion, heart failure, arrhythmia, superior vena cava compression syndrome, and fever [Lowenthal 1988]. Once the cardiac conduction system was involved, ECG showed high AV block, atrial fibrillation, atrial flutter, low voltage and T wave inversion or low level [Ito 2005]; timely intervention can improve the postoperative recovery and long-term prognosis [Petrich 2011]. The tumor of this case was located in the right atrium. The patient was immunocompetent, and HIV tests were negative. Chest tightness and shortness of breath are the main symptoms, whereas ECG showed atrial flutter and third degree AV block. Right atrial tumor excision and cardiac pacemaker implantation were performed during the operation, which can prevent the perioperative malignant arrhythmia and improve the long-term prognosis.

PCL is difficult to diagnose and sometimes depends on invasive tests. Even in some cases, autopsy is necessary for final diagnosis. Transesophageal echocardiography (TEE) was the main less invasive and sensitive mechanism for examining the chambers of the heart and pericardium, especially for cardiac tumors. This can accurately detect tumor location, size, mobility, degree of invasion, and friability. Its sensitivity depends on the size of the tumors and the ultrasound image of each patient. CT and MRI not only allow the diagnosis of polypoid, ill-defined, infiltrating tumors, but also provide information about the type of tumor. The use of PET-CT not only displays the proliferation and metabolism of the tumor more effectively, but also indicates the staging, curative effect, and prognosis of cardiac lymphoma more accurately [Minamimoto 2011; Yoshihara 2013; Fukunaga 2018]. The lymphoma cells may be found through pericardiocentesis or chest drainage in patients with pericardial or pleural effusion. However, histopathological examination is still necessary to make a definite diagnosis. This patient was diagnosed as
DLBCL by pathological biopsy. The tumor cells expressed both $\mathrm{Bcl}-2$ and c-MYC proteins and also $\mathrm{Ki}-67$ (+, about 90\%), with Ki-67 having high proliferative activity. Regular PET-CT examination after chemotherapy guided the further treatment and prognosis.

The prognosis of PCL is poor, especially in patients with myocardial infiltration [Wang 2014]. Chemotherapy, radiotherapy, and surgery are the main therapies at present. Surgical operation not only applies to the mechanical obstruction, but also leads to identification of the histopathological types of the mass. However, surgical treatment by itself cannot improve the prognosis because of the infiltrative growth of PCL [Zhong 2013]. DLBCL is the most common type of PCL, and with high invasiveness; so early systemic chemotherapy after a definite diagnosis is the most effective treatment for the disease [Petrich 2011]. Some reports showed that chemotherapy combined with radiotherapy may be helpful to improve the survival rate of patients with PCL [Ceresoli 1997]. R-CHOP or R-EPOCH is currently recommended for patients with DLBCL, and chemotherapy should not be less than 6 courses. If the disease is progressing, autologous or even allogeneic hematopoietic stem cell transplantation should be considered after PR or CR [Nonami 2007]. In this case the tumor had already infiltrated the myocardium, so the tumor was resected as much as possible, and a pacemaker was implanted to correct the arrhythmia during the operation. The pathological type of the tumor was DLBCL, which involved the heart and bilateral kidneys and expressed both Bcl-2 and c-MYC proteins and also Ki-67 (+, about 90\%), with Ki-67 having high proliferative activity. Autologous peripheral blood stem cell transplantation was performed after 6 courses of R-CHOP and 1 course of R-EPOCH, followed by PET-CT evaluation is CR. The patient remained free of lymphoma for 1 year.

\section{CONCLUSIONS}

PCL is a rare malignant tumor of the heart. Although its nonspecific clinical manifestations are difficult to diagnose, it is still possible to detect the tumor in early stage and give surgery or heart transplantation in time, with the development of modern imaging techniques, biopsy, and clinical thinking. The early high-dose, systematic chemotherapy in accordance with postoperative pathological results can significantly improve the prognosis of and the quality of life of patients.

\section{ACKNOWLEDGMENTS}

Funding: Not applicable.

Authors' contributions: S.L., C.R., R.W., Y.W.

Ethical approval and consent to participate: Not applicable.

Consent for publication: Consent given.

Availability of supporting data: Not applicable.

Competing interests: I certify that there is no conflict of interest with any financial organization regarding the material discussed in the manuscript. 
Other: I am deeply indebated to all other writers and teachers in translation studies for their direct and indirect help to me. Special thanks should go to my colleagues who provide valuable pictures and data. Finally, I am indebted to my families for their continous support and encouragement.

\section{REFERENCES}

Baikoussis NG, Papakonstantinou NA, Dedeilias P, et al. 2015. Cardiac tumors: a retrospective multicenter institutional study. J BUON 20(4):1115-23.

Basso C, Valente M, Thiene G, eds. c2013. Cardiac tumor pathology. New York: Humana Press.

Ceresoli GL, Ferreri AJ, Bucci E, Ripa C, Ponzoni M, Villa E. 1997. Primary cardiac lymphoma in immunocompetent patients: diagnostic and therapeutic management. Cancer 80(8):1497-506.

Coiffier B, Lepage E, Briere J, et al. 2002. CHOP chemotherapy plus rituximab compared with $\mathrm{CHOP}$ alone in elderly patients with diffuse large-B-cell lymphoma. N Engl J Med 346(4):235-42.

Curtsinger CR, Wilson MJ, Yoneda K. 1989. Primary cardiac lymphoma. Cancer 64(2):521-5.

Fukunaga H, Tatewaki Y, Mutoh T, et al. 2018. A case of low-grade primary cardiac lymphoma with pericardial effusion diagnosed by combined 18F-fluorodeoxyglucose positron emission tomography and computed tomography (FDG-PET/CT) imaging and effusion cytology. Am J Case Rep 19:292-5.

Ito M, Tsuchiyama J, Chinushi M, Kodama M, Aizawa Y. 2005. Transient giant negative $\mathrm{T}$ waves associated with cardiac involvement of diffuse large B-cell lymphoma. Circulation 112(20):e322-3.

Lam KY, Dickens P, Chan AC. 1993. Tumors of the heart. A 20 year experience with a review of 12,485 consecutive autopsies. Arch Pathol Lab Med 117(10):1027-31.
Lowenthal DA, Straus DJ, Campbell SW, Gold JW, Clarkson BD, Koziner B. 1988. AIDS-related lymphoid neoplasia. The Memorial Hospital experience. Cancer 61(11):2325-37.

Minamimoto R, Morooka M, Kubota K, et al. 2011. Value of FDG-PET/ CT using unfractionated heparin for managing primary cardiac lymphoma and several key findings. J Nucl Cardiol 18(3):516-20.

Nonami A, Takenaka K, Kamezaki K, et al. 2007. Successful treatment of primary cardiac lymphoma by rituximab-CHOP and high-dose chemotherapy with autologous peripheral blood stem cell transplantation. Int J Hematol 85(3):264-6.

Petrich A, Cho SI, Billett H. 2011. Primary cardiac lymphoma: an analysis of presentation, treatment, and outcome patterns. Cancer 117(3):581-9.

Svec A, Rangaiah M, Giles M, Jaksa R, McAulay KA. 2012. EBV+ diffuse large B-cell lymphoma arising within atrial myxoma. An example of a distinct primary cardiac EBV+ DLBCL of immunocompetent patients. Pathol Res Pract 208(3):172-6.

Tanaka PY, Atala MM, Pereira J, Caterino-de-Araujo A. 2009. Primary effusion lymphoma with cardaic involvement in HIV positive patientcomplete response and long survival with chemotherapy and HAART. J Clin Virol 44(1):84-85.

Travis WD, Brambilla E, Burke AP, Marx A, Nicholson AG, eds. 2015. WHO classification of tumours of the lung, pleura, thymus and heart. Lyon: IARC Press.

Wang Q, Zhu J. 2014. Diagnosis and treatment of primary cardiac lymphoma in China. J Guangxi Med 36(5):671-3.

Yoshihara S, Naito M, Tanioka F, Matsunaga M. 2013. A case of primary cardiac lymphoma: in vivo imaging and pathologic correlation. Eur Heart J Cardiovasc Imaging 14(10):1027.

Zaharia L, Gill PS. 1991. Primary cardiac lymphoma. Am J Clin 0ncol 14(2):142-5.

Zhong L, Yang S, Lei K, Jia Y. 2013. Primary cardiac lymphoma: a case report and review of the literature. Chin German J Clin Oncol 12(1):43-5. 